\title{
Detecting sentinel lymph nodes of varying site in endome- trial cancer patients
}

\author{
Hwajung Lee, Kyung-Jin Min, Sanghoon Lee, Jin Hwa Hong, Jae Kwan Lee, Nak-Woo Lee, Jae Yun Song \\ Department of Obstetrics and Gynecology, Korea University College of Medicine, Seoul, Korea
}

Objective: We report three cases of sentinel lymph node identification in endometrial cancer patients that each present different location of pelvic sentinel lymph node.

Methods: The da Vinci Xi model was used by single surgeon to perform staging operation with sentinel lymph node biopsy. All three patients were pre-operatively diagnosed with stage I endometrial cancer and showed no distinct lymph node enlargement on pelvic magnetic resonance imaging or computed tomography. A total of $4 \mathrm{~mL}$ of indocyanine green (ICG) was injected at 3 and 9 o'clock direction on cervix, and we biopsied the identified sentinel lymph nodes for pathologic confirmation.

Results: The sentinel lymph nodes were detected from various locations of pelvis even though process of sentinel lymph node detection were identical for each surgery. All lymph nodes were found to be negative of malignancy and there was no post-operative complication.

Conclusion: The sentinel lymph node detection during robotic surgery in endometrial cancer patient is widely accepted procedure in gynecologic oncology field. However, it should be noted that sentinel lymph nodes can be detected from varying locations of the pelvic cavity, not necessarily near-the-injection-site.

Key Words: Sentinel lymph node; Robotic surgery; Indocyanine green

\section{Video related to this article}

The video related to this article can be found online at 10.36637/grs.2021.00031.

\section{Conflict of interest}

No potential conflict of interest relevant to this article was reported.

- Received: March 23, 2021 • Accepted: March 24, 2021

- Correspondening author: Jae Yun Song

Department of Obstetrics and Gynecology, Korea University Anam Hospital, Korea University College of Medicine, 73 Goryeodae-ro, Seongbuk-gu, Seoul 02841, Korea

E-mail: sjyuni105@gmail.com

This is an Open Access article distributed under the terms of the Creative Commons Attribution Non-Commercial License (http://creativecommons.org/ licenses/by-nc/4.0) which permits unrestricted non-commercial use, distribution, and reproduction in any medium, provided the original work is properly cited. 
Varying sites of sentinel lymph node $\mid \mathrm{Lee} \mathrm{H}$, et al.

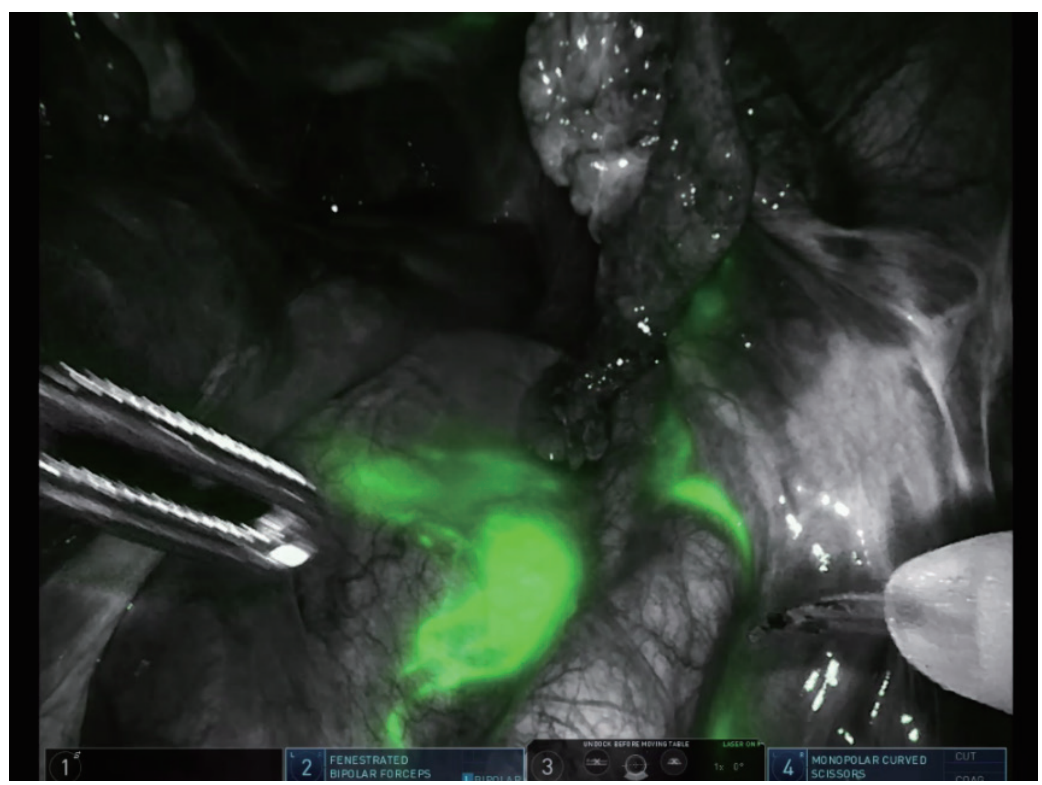

Video 1. 